\title{
BMW I TUM I LEHRSTUHL FÜR SYSTEMISCHE FAHRZEUGEFFIZIENZ
}

Die BMW Group und die Technische Universität München (TUM) haben die Einrichtung eines Stiftungslehrstuhls für systemische Fahrzeugeffizienz vereinbart. Mit der Stiftung möchte der Automobilhersteller Forschung und Lehre in diesem Querschnittsthema des Automobilbaus stärken. Kürzlich unterzeichneten Dr. Klaus Draeger, Vorstandsmitglied der BMW AG, und TUM-Präsident Wolfgang A. Herrmann den Vertrag mit einer Laufzeit von fünf Jahren und einer Stiftungssumme von 5,1 Millionen Euro.

BMWs Motivation, den neuen Lehrstuhl für systemische Fahrzeugeffizienz zu gründen, liegt in der steigenden Nachfrage nach nachhaltigen Mobilitätslösungen und effizienteren technischen Systemen. Diese Nachfrage werde durch knapper werdende Ressourcen und die zunehmende Urbanisierung bedingt, und ihr sollte im Automobilbau bereits in der universitären Ausbildung Rechnung getragen werden, erklärte der Münchener Automobilhersteller.

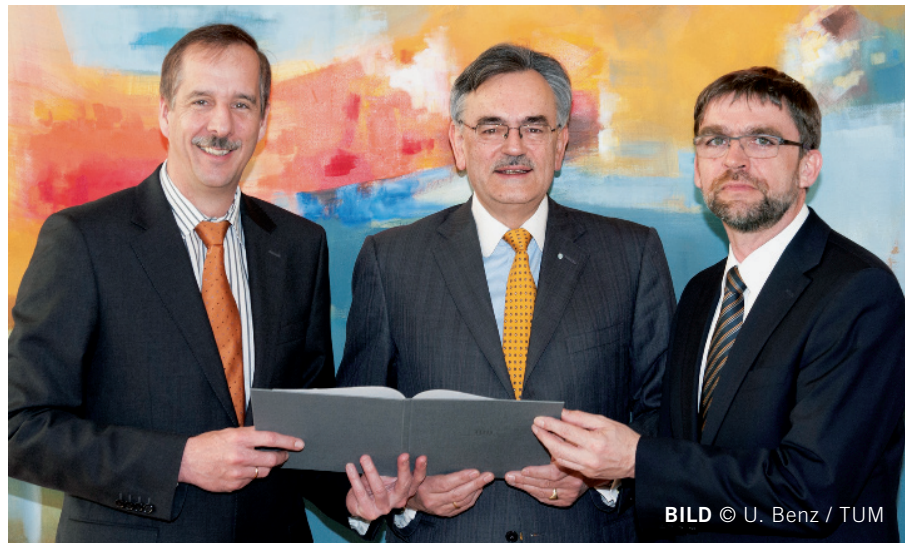

Von links nach rechts: Dr.-Ing. Klaus Draeger (BMW), Prof. Wolfgang A. Herrmann (TUM), Dr. Albin Dirndorfer (BMW)
Laut Draeger fordere die Ausrichtung auf Effizienz das Know-how von Ingenieuren, die sich schon in ihrer Ausbildung mit Effizienz vertraut gemacht haben.

Die Entscheidung der BMW Group, den Stiftungslehrstuhl an der Technischen Universität München einzurichten, gründet auf der seit Jahrzehnten bewährten und erfolgreichen Zusammenarbeit. „Die TUM ist eine international anerkannte Forschungsuniversität, die viele unserer Ingenieure und Naturwissenschaftler ausgebildet hat“, so Draeger. „Sie hat sich einen hervorragenden Ruf in Zukunftsbereichen wie Automobilinformatik, Leichtbau und Elektromobilität erarbeitet."

\section{DHBW I NEUER MASTERSTUDIENGANG AUTOMOTIVE SYSTEMS ENGINEERING}

Die Duale Hochschule Baden-Württemberg (DHBW) baut mit neuen Masterstudiengängen ihre Weiterbildungsangebote aus: Berufsbegleitend kann nun der Studiengang Automotive Systems Engineering - Green Technology belegt werden. Das Angebot wird gemeinsam von sieben DHBW-Standorten angeboten und richtet sich an junge Fachund Führungskräfte, die bereits erste Berufserfahrung gesammelt haben.

Mit der Frage, wie angesichts von Klimawandel und Energiewende die Antriebskonzepte der Zukunft aussehen, werden sich Studierende des Masterstudiengangs Automotive Systems Engineering - Green Technology künftig auseinandersetzen. Das neue Studienangebot wird gemeinsam mit den weiteren neuen Masterstudiengängen Informatik, Maschinenbau und Wirtschaftsingenieurwesen zum Wintersemester 2012/13 an der DHBW eingerichtet. Bewerbungen sind ab sofort möglich. Interessenten müssen neben einem ersten Studienabschluss mindestens ein Jahr Berufserfahrung in qualifizierter Position vorweisen.

Die Inhalte des Studiengangs Automotive Systems Engineering - Green Technology

zielen auf die Entwicklung ökologisch nachhaltiger Fahrzeuge und Fahrzeugkomponenten. Der Fokus liegt auf der Reduzierung der Schadstoffemission und der Energieoptimierung. Im Blickpunkt steht die Elektromobilität mit neuen Konzepten für elektrische Antriebe und Energiespeicher sowie Lösungsansätzen für Energiemanagement. Auf der Basis eines technischen Bachelorstudiengangs bietet der Masterstudiengang eine zusätzliche und erweiternde Qualifizierung für die Automobilindustrie. Es werden fachliche, persönliche und soziale Kompetenzen vermittelt. Sowohl zukünftige Experten als auch angehende Führungskräfte werden angesprochen. Abgeschlossen werden kann das Studium mit dem Master of Engineering (M.Eng.) Das Studium ist berufsbegleitend ausgelegt und dauert vier Semester. Gelehrt wird teilweise in deutscher und in englischer Sprache.

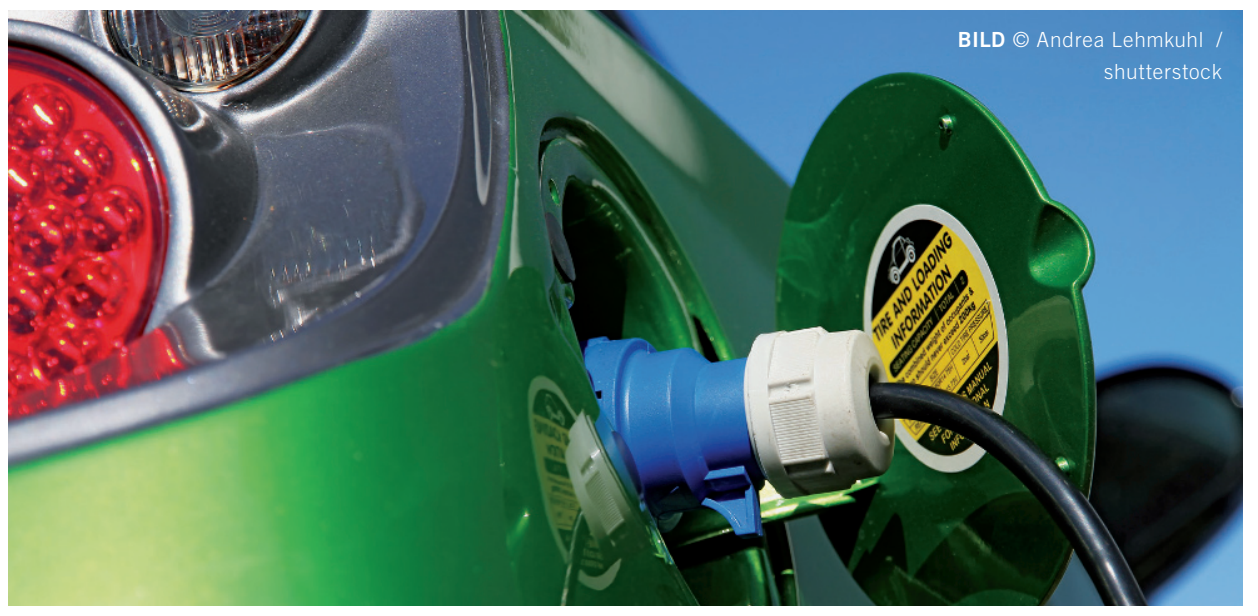

